\title{
On The Spectral Properties of Non- Self-Adjoint Elliptic Differential Operators in Hilbert space
}

\author{
Reza Alizadeha ${ }^{a}$ Ali Sameripour ${ }^{a}$ \\ ${ }^{a}$ Department of Mathematics, Lorestan University, Khoramabad, Iran.
}

\begin{abstract}
The non-self-adjoint operators appear in many branches of science, from kinetic theory and quantum mechanics to linearizations of equations of mathematical physics. Non-self-adjoint operators are usually difficult to study because of the lack of general spectral theory. In this paper, our aim is to study the resolvent and the spectral properties of a class of non-self-adjoint differential operators.
\end{abstract}

Keywords: resolvent, asymptotic spectrum, distribution of eigenvalues, non-self-adjoint differential operator.

2010 MSC: 35P20, 35J99, 35P99.

\section{Introduction}

Let $\Omega$ be a bounded domain in $R^{n}$ with smooth boundary $\partial \Omega$ (i.e., $\partial \Omega \in C^{\infty}$ ). We introduce the weighted Sobolev space $\mathcal{H}=W_{2, \alpha}^{2}(\Omega)$ as the space of complex value functions $u(x)$ defined on $\Omega$ with finite norm:

$$
|u|_{+}=\left(\sum_{i=1}^{n} \int_{\Omega} \sigma^{2 \alpha}(x)\left|u_{x_{i}}^{\prime}(x)\right|^{2} d x+\int_{\Omega}|u(x)|^{2} d x\right)^{1 / 2}
$$

where $0 \leq \alpha<1$, and Here $\sigma(x)$ is weighted function, $\mu(x) \in C^{2}(\bar{\Omega})$. We denote by $\stackrel{\circ}{\mathcal{H}}$ the closure of $C_{0}^{\infty}(\Omega)$ in $\mathcal{H}$ with respect to the above norm. i.e., $\mathcal{H}$ is the closure of $C_{0}^{\infty}(\Omega)$ in $W_{2, \alpha}^{2}(\Omega)$. The notion $C_{0}^{\infty}(\Omega)$ stands for the space of infinitely differentiable functions with compact support in $\Omega$. In this paper we investigate the

Email addresses: Alizadeh.re@fs.lu.ac.ir (Reza Alizadeh), asameripour@yahoo.com (Ali Sameripour) 
spectral properties, in particular we estimate the resolvent of a non-self adjoint elliptic differential operator of type $(A u)(x)=-\sum_{i, j=1}^{n}\left(\sigma^{2 \alpha}(x) a_{i j}(x) \mu(x) u_{x_{i}}^{\prime}(x)\right)_{x_{j}}^{\prime}$ acting in Hilbert space $H=L^{2}(\Omega)$. Now, we need to extend its domain as follows:

$$
D(A)=\left\{y \in \stackrel{\circ}{H} \cap W_{2, l o c}^{2}(\Omega): \sum_{i, j=1}^{n}\left(\sigma^{2 \alpha} a_{i j} \mu y_{x_{i}}^{\prime}\right)_{x_{j}}^{\prime} \in H\right\}
$$

(see cite2, [3]) where the local space $W_{2, l o c}^{2}(\Omega)$ is the class of the functions $u(x)(x \in \Omega)$ in this form

$$
W_{2, l o c}^{2}(\Omega)=\left\{u(x): \sum_{i=0}^{2} \int_{J}\left|u^{(i)}(x)\right|^{2} d x<\infty, \quad J \subset \Omega, \text { open }\right\}
$$

Here $\sigma(x)$ is weighted function, $0 \leq \alpha<1, \mu(x) \in C^{2}(\bar{\Omega}), \quad a_{i j}(x) \in C^{2}(\bar{\Omega})$,

$a_{i j}(x)=a_{j i}(x)$ and the functions $a_{i j}(x)$ satisfies the uniformly elliptic condition, i.e., there exists $c>0$ such that: $c|s|^{2} \leq \sum_{i, j=1}^{n} a_{i j}(x) s_{i} \overline{s_{j}}$ where $s=\left(s_{1}, \ldots, s_{n}\right) \in \mathbf{C}^{n}, \quad x \in \Omega$. Assume that $\mu(x) \in \mathbf{C} \backslash \Phi, \forall x \in \bar{\Omega}$ where $\Phi=\{z \in \mathbf{C}:|\arg z| \leq \varphi\}, \varphi \in(0, \pi)$ (i.e., the value of $\mu(x)$ lie on the complex plane and outside of the closed angle $\Phi)$. To get a feeling for the history of the subject under study, refer to our earlier papers [9], [10]. Indeed this paper was written in continuing with earlier our papers, the paper is sufficiently more general than earlier our papers.

\section{Main Results}

Theorem 2.1. Let $(A u)(x)=-\sum_{i, j=1}^{n}\left(\sigma^{2 \alpha}(x) a_{i j}(x) \mu(x) u_{x_{i}}^{\prime}(x)\right)_{x_{j}}^{\prime}$ acting in Hilbert space $H=L^{2}(\Omega)$ with Dirichlet-type boundary conditions. Here $\sigma(x)$ is weighted function, $0 \leq \alpha<1, \mu(x) \in C^{2}(\bar{\Omega})$, Choose a closed sector $S \subset \Phi$ with its vertex at zero (for more explain see [8]), such that $S \cap R_{+}=\emptyset$. Let the complex function $\mu(x)$ satisfies the following conditions

$$
\begin{gathered}
\mu(x) \in C^{1}(\bar{\Omega}), \mu(x) \in \mathbf{C} \backslash S, \quad(\forall x \in \bar{\Omega}), \\
\left|\arg \left\{\mu\left(x_{1}\right) \mu^{-1}\left(x_{2}\right)\right\}\right| \leq \frac{\pi}{8}, \quad\left(\forall x_{1}, x_{2} \in \bar{\Omega}\right) .
\end{gathered}
$$

Then, for sufficiently large in modulus $\lambda \in S$, the inverse operator $(A-\lambda I)^{-1}$ exists and is continuous in $H$, and the following estimates are valid

$$
\begin{gathered}
\left\|(A-\lambda I)^{-1}\right\| \leq M_{S}|\lambda|^{-1}\left(\lambda \in S,|\lambda|>C_{S}\right), \\
\left\|\sigma^{\alpha} \frac{\partial}{\partial x_{i}}(A-\lambda I)^{-1}\right\| \leq M_{S}^{\prime}|\lambda|^{-\frac{1}{2}}\left(\lambda \in S,|\lambda|>C_{S}\right), \\
\text { for } i=1, \ldots, n
\end{gathered}
$$

where $M_{S}, C_{S}>0$ are sufficiently large numbers depending on $S$. The symbol $\|$.$\| stands for the norm of a$ bounded arbitrary operator $T$ in $H$.

Proof. Here, to establish Theorem 2.1, we will first prove the assertion of Theorem 2.1 together with estimate (2.3). So, as in Section 1 for the closed extension the operator $A$ (for more explain see chapter 6 of [8]), we need to extend its domain to the closed set

$$
D(A)=\left\{y \in \stackrel{\circ}{H} \cap W_{2, l o c}^{2}(\Omega): \sum_{i, j=1}^{n}\left(\sigma^{2 \alpha} a_{i j} \mu y_{x_{i}}^{\prime}\right)_{x_{j}}^{\prime} \in H\right\}
$$

Let the operator $A$, now satisfy (2.1), (2.2). Then there exists a complex number $Z \in C$ (noticed that we can take $Z=e^{i \gamma}$, for a fix real $\left.\gamma \in(-\pi, \pi]\right)$, then we have $\left|Z=e^{i \gamma}\right|=1$, and so

$$
c^{\prime} \leq \operatorname{Re}\{Z \mu(x)\}, \quad c^{\prime}|\lambda| \leq-\operatorname{Re}\{Z \lambda\}, \quad c^{\prime}>0(\forall x \in \bar{\Omega}, \lambda \in \Phi) .
$$


In view of the uniformly elliptic condition, we have

$$
c|s|^{2}=c \sum_{i=1}^{n}\left|s_{i}\right|^{2} \leq \sum_{i, j=1}^{n} a_{i j}(x) s_{i} \overline{s_{j}},\left(c>0, s=\left(s_{1}, \ldots, s_{n}\right) \in \mathbf{C}^{n}, x \in \Omega\right)
$$

take $s_{i}=y_{x_{i}}^{\prime}$ implies that $c \sum_{i=1}^{n}\left|y_{x_{i}}^{\prime}(x)\right|^{2} \leq \sum_{i, j=1}^{n} a_{i j}(x) y_{x_{i}}^{\prime}(x) \overline{y_{x_{j}}^{\prime}(x)}$. From this, and according to $c^{\prime} \leq$ $\operatorname{Re}\{Z \mu(x)\}$ in (2.4), we then multiply these two positive relations with each other implies that

$$
c_{1} \sum_{i=1}^{n}\left|y_{x_{i}}^{\prime}(x)\right|^{2} \leq \operatorname{Re} Z \mu(x) \sum_{i, j=1}^{n} a_{i j}(x) y_{x_{i}}^{\prime}(x) \overline{y_{x_{j}}^{\prime}(x)} \text {. for } y \in D(A)
$$

Multiply both sides of the latter relation by the positive term $\sigma^{2 \alpha}(x)$, and then integrate from both sides, we will have

$$
c_{1} \sum_{i=1}^{n} \int_{\Omega} \sigma^{2 \alpha}(x)\left|y_{x_{i}}^{\prime}(x)\right|^{2} d x \leq \operatorname{Re} Z \sum_{i, j=1}^{n} \int_{\Omega} \sigma^{2 \alpha}(x) a_{i j}(x) \mu(x) y_{x_{i}}^{\prime}(x) \overline{y_{x_{j}}^{\prime}(x)} d x .
$$

Now by applying the integration by parts, and using Dirichlet-type condition, then the right sides of the latter relation without multiple $R e Z$ becomes:

$$
\begin{gathered}
\sum_{i, j=1}^{n} \int_{\Omega} \sigma^{2 \alpha}(x) a_{i j}(x) \mu(x) y_{x_{i}}^{\prime}(x) \overline{y_{x_{j}}^{\prime}(x)} d x \\
=-\sum_{i, j=1}^{n} \int_{\Omega}\left(\sigma^{2 \alpha}(x) a_{i j}(x) \mu(x) y_{x_{i}}^{\prime}(x)\right)_{x_{j}}^{\prime} \bar{y}(x) d x \\
=\left(-\sum_{i, j=1}^{n}\left(\sigma^{2 \alpha}(x) a_{i j}(x) \sigma(x) y_{x_{i}}^{\prime}(x)\right)_{x_{j}}^{\prime}, y(x)\right)=(A y, y) .
\end{gathered}
$$

Since $(A y)(x)=-\sum_{i, j=1}^{n}\left(\sigma^{2 \alpha}(x) a_{i j}(x) \mu(x) u_{x_{i}}^{\prime}(x)\right)_{x_{j}}^{\prime}$. Here, the the symbol (,) denotes the inner product in $H$. Notice that the above equality in (2.6) obtains by the well known theorem of the $\mathrm{m}$-sectorial operators which are closed by extending its domain to the closed domain in $\mathcal{H}$. These operators are associated with the closed sectorial bilinear forms that are densely defined in $\mathcal{H}$ (for more explanation see the well known Theorem 2.1. chapter 6 of [8]). The reason we extend the domain of the operator $A$ to the closed domain in space $\mathcal{H}$, above is now specified.

therefore

$$
c_{1} \sum_{i=1}^{n} \int_{\Omega} \sigma^{2 \alpha}(x)\left|y_{x_{i}}^{\prime}(x)\right|^{2} d x \leq \operatorname{Re} Z(A y, y)
$$

from (2.4) we have: $c^{\prime}|\lambda| \leq-\operatorname{Re}\{Z \lambda\}, c^{\prime}>0, \forall \lambda \in \Phi$. Multiply this inequality by $\int_{\Omega}|y(x)|^{2} d x=(y, y)=$ $\|y\|^{2}>0$. It follows that $c^{\prime}|\lambda| \int_{\Omega}|y(x)|^{2} d t \leq-\operatorname{Re}\{Z \lambda\}(y, y)$. From this and the above inequality we will have

$$
\begin{aligned}
c_{1} \sum_{i=1}^{n} \int_{\Omega} \sigma^{2 \alpha}(x)\left|y_{x_{i}}^{\prime}(x)\right|^{2} d x+c^{\prime}|\lambda| \int_{\Omega}|y(x)|^{2} d x & \leq \operatorname{Re}\{Z(A y, y)-Z \lambda(y, y)\} \\
& =\operatorname{Re}\{Z((A-\lambda I) y, y)\} \\
& \leq\|Z\|\|y\|\|(A-\lambda I) y\| \\
& =\|y\|\|(A-\lambda I) y\| ; \quad(2.7)
\end{aligned}
$$


i.e.,

$$
c_{1} \sum_{i=1}^{n} \int_{\Omega} \sigma^{2 \alpha}(x)\left|y_{x_{i}}^{\prime}(x)\right|^{2} d x+c^{\prime}|\lambda| \int_{\Omega}|y(x)|^{2} d x \leq\|y\|\|(A-\lambda I) y\| .
$$

Since $c_{1} \sum_{i=1}^{n} \int_{\Omega} \sigma^{2 \alpha}(x)\left|y_{x_{i}}^{\prime}(x)\right|^{2} d x$ is positive. We will have either $c^{\prime}|\lambda|\|y(x)\|^{2}=|\lambda| \int_{\Omega}|y(x)|^{2} d x \leq$ $\|y\|\|(A-\lambda I) y\|$. Or

$$
|\lambda|\|y(x)\| \leq M_{S}\|(A-\lambda I) y\| .
$$

This inequity ensures that the operator $(A-\lambda I)$ is one to one, which implies that $\operatorname{ker}(A-\lambda I)=0$. Therefore the inverse operator $(A-\lambda I)^{-1}$ exists, and its continuity follows from the proof of the estimate (2.3) of Theorem 2.1. To prove (2.3), we set $v=(A-\lambda I)^{-1} f, f \in H$ in (2.7) implies that

$$
|\lambda| \int_{\Omega}\left|(A-\lambda I)^{-1} f\right|^{2} d x \leq M_{S}\left\|(A-\lambda I)^{-1} f\right\|\left\|(A-\lambda I)(A-\lambda I)^{-1} f\right\| .
$$

Since $(A-\lambda I)(A-\lambda I)^{-1} f=I(f)=f$. Then

$$
|\lambda| \int_{\Omega}\left|(A-\lambda I)^{-1} f\right|^{2} d x \leq M_{S}\left\|(A-\lambda I)^{-1} f\right\||f| .
$$

So

$$
|\lambda|\left\|(A-\lambda I)^{-1}(f)\right\|^{2} \leq M_{S}\left\|(A-\lambda I)^{-1}(f)\right\||f| .
$$

Which this implies that $|\lambda|\left\|(A-\lambda I)^{-1}(f)\right\| \leq M_{S}|f|$. Since $\lambda \neq 0$. Then $\left\|(A-\lambda I)^{-1}(f)\right\| \leq M_{S}|\lambda|^{-1}|f|$; i.e., $\left\|(A-\lambda I)^{-1}\right\| \leq M_{S}|\lambda|^{-1}$. This estimate completes the proof of the assertion of Theorem 2.1 together with the estimate (2.3). Now, we start to prove the estimate (2.4) of Theorem 2.1 As in the above argument, we drop the positive term $c^{\prime}|\lambda| \int_{\Omega}|y(x)|^{2} d x$ from

$$
c_{1} \sum_{i=1}^{n} \int_{\Omega} \sigma^{2 \alpha}(x)\left|y_{x_{i}}^{\prime}(x)\right|^{2} d x+c^{\prime}|\lambda| \int_{\Omega}|y(x)|^{2} d x \leq\|y\|\|(A-\lambda I) y\| .
$$

It follows that

$$
c_{1} \sum_{i=1}^{n} \int_{\Omega} \sigma^{2 \alpha}(x)\left|y_{x_{i}}^{\prime}(x)\right|^{2} d x \leq\|y\|\|(A-\lambda I) y\| .
$$

Eminently,

$$
c_{1}\left\|\sigma^{\alpha} \frac{\partial}{\partial x_{i}}(A-\lambda I)^{-1} f\right\|^{2} \leq\|y\|\|(A-\lambda I) y\| .
$$

Set $y=(A-\lambda I)^{-1} f, f \in H$ in the latter relation, and proceeding by similar calculation as in the proof (2.3) we then obtain:

$$
c_{1}\left\|\sigma^{\alpha} \frac{\partial}{\partial x_{i}}(A-\lambda I)^{-1} f\right\|^{2} \leq\left\|(A-\lambda I)^{-1} f\right\|\left\|(A-\lambda I)(A-\lambda I)^{-1} f\right\| .
$$

Since $(A-\lambda I)(A-\lambda I)^{-1} f=I(f)=f$, then

$$
c_{1}\left\|\sigma^{\alpha} \frac{\partial}{\partial x_{i}}(A-\lambda I)^{-1} f\right\|^{2} \leq\left\|(A-\lambda I)^{-1}\right\| f \|^{2},
$$

consequently, by (2.3) this implies that

$$
c_{1}\left\|\sigma^{\alpha} \frac{\partial}{\partial x_{i}}(A-\lambda I)^{-1} f\right\|^{2} \leq M_{S}|\lambda|^{-1}\|f\|^{2}
$$

to this end we will have

$$
\left\|\sigma^{\alpha} \frac{\partial}{\partial x_{i}}(A-\lambda I)^{-1}\right\| \leq M_{S}^{\prime}|\lambda|^{-\frac{1}{2}} .
$$

Thus, here the proof of the estimate (2.4) is finished; i.e., this completes the proof of Theorem 2.1 


\section{References}

[1] M.S.Agranovich, Elliptic operators on compact manifolds,I.Itogi Nauki I Tekhniki: Sovremennye Problemy Mat :Fundamental'nye Napravleniya Val.63, VINITI, Moskow.1990, PP.5-129 (Russian)

[2] K. Kh. Boimatov and A. G. Kostyuchenko, Distribution of eigenvalues of second-order non-selfadjoint differential operators, Vest. Mosk. Gos. Univ., Ser. I, Mat. Mekh, No. 3, 1990, pp. 24-31 (Russian).

[3] K. Kh. Boimatov, Asymptotic behaviour of the spectra of second-order non-selfadjoint systems of differential operators, Mat. Zametki, Vol. 51, No. 4, 1992, pp. 6-16, (Russian).

[4] K. Kh. Boimvatov, Spectral asymptotics of nonselfadjoint degenerate elliptic systems of differential operators Dokl. Akad. Nauk. Rossyi, Vol. 330, No.6, 1993,(Russian); (English transl. In Russian Acad.Sci.Dokl. Math. Vol.47, 1993, N3, PP.545$553)$

[5] K. Kh. Boimvatov, Separation theorems, weighted spaces and there applications. Trudy Mat. Inst. Steklov. Vol.170,1984, P.37-76,(Russian) (English transl. in Pros.Steklov. Inst. Math. 1987, N1 (170)

[6] K. Kh. Boimatov, Spectral asymptotics of differential and pseudo-differential operators Part.2, Trudy sem.Ptrosk.V.10.1984.P.78-106, Russian, (English transl. In Soviet Math.V.35, N.5, 1986)

[7] I. C. Gokhberg and M. G. Krein, Introduction to the Theory of linear non-selfadjoint operators in Hilbert space, English transl. Amer. Math. Soc., Providence, R. I. 1969.

[8] T. Kato, Perturbation Theory for Linear Operators, Springer, New York, 1966.

[9] A. Sameripour and K. Seddigh, Distribution of the eigenvalues non-selfadjoint elliptic systems that degenerated on the boundary of domain, (Russian)Mat. Zametki 61(1997), no,3, 463-467 translation in Math. Notes 61(1997) no,3-4. 379-384 (Reviewer: Gunter Berger) 35P20(35J55)

[10] A. Sameripour, On the Distribution of eigenvalues of degenerate elliptic differetial oparetors far from self adjoint ones for general boundary conditions,Ann. Sci.Math.Quebec 27 (2003), no.1,67-89. (Reviewer:Alexi Yu.Konstantinov) 34L10 (34L05 34L20 35P05 47E05)

[11] A. A. Shkalikov, Tauberian type theorems on the distribution of zeros of holomorphic functions, Matem. Sbornik Vol. 123 (165) 1984, No. 3, pp. 317-347; English transl. in Math. USSR-sb. 51, 1985.

[12] I.L. Vulis and M. Z. Solomyak, Spectral asymptotics of degenerate elliptic staklov problem, vestn. Leningr. Univ., No:19, 148-150(1973)

[13] I.L. Vulis and M. Z. Solomyak, Spectral asymptotics of degenerate elliptic operators, Dokl.Akad. Nauk. SSSR,207,NO.2, 262-265 (1972) 